\title{
SOLUTION OF ENERGY TRANSPORT EQUATION WITH VARIABLE MATERIAL PROPERTIES BY BEM
}

\author{
J. RAVNIK ${ }^{1}$, L. SKERGET ${ }^{1}$, J. TIBAUT ${ }^{1} \&$ B.W. YEIGH ${ }^{2}$ \\ ${ }^{1}$ University of Maribor, Faculty of Mechanical Engineering, Smetanova ulica 17, SI-2000 Maribor, Slovenia. \\ ${ }^{2}$ University of Washington, 18115 Campus Way NE Bothell WA 98011-8246, USA.
}

\begin{abstract}
In this paper, we derive a boundary-domain integral formulation for the energy transport equation under the assumption that the fluid properties, through which the energy is transported by diffusion and convection, are spatially and temporally changing. The energy transport equation is a second- order partial differential equation of a diffusion-convection type, with the fluid temperature as the independent variable. The presented formulation does not require a calculation of the temperature gradient, thus it is, for a known fluid velocity field, linear.

The final boundary-domain integral equation is discretized using a domain decomposition approach, where the equation is solved on each sub-domain, while subdomains are joined by compatibility conditions. The validity of the method is checked using several analytical examples. Convergence properties are studied yielding that the proposed discretization technique is second-order accurate.

The developed method is used to simulate flow and heat transfer of nanofluids, which exhibit properties that depend on the solid particle concentration. A Lagrange-Euler approach is used.

Keywords: boundary element method, energy transport equation, nanofluids, variable material properties.
\end{abstract}

\section{INTRODUCTION}

Many natural phenomena involve energy transfer, which is governed by the diffusion and convection transport processes. In nature and for most engineering purposes, heat transfer occurs in environments, where the velocity of the fluid changes within the domain in question. Fluid properties, such as density, specific heat and heat conductivity are usually considered as constant.

However, there are examples, where changes in fluid material properties must be considered. One example is a case, where large temperature differences are present in the simulation domain. Since material properties are temperature depended, these must be considered. Another example are nanofluids. These are suspensions of nanometer-sized particles in a base liquid. The properties of the suspension (when modeled as a single-phase liquid with modified properties) depend on the concentration of the particles, which in turn depends on the flow field.

Solution of the diffusion-convection partial differential equation is a challenging task. Many numerical algorithms have been proposed. In terms of the boundary element method by using the diffusion-convection fundamental solution, the problem can (at least for constant velocity field and constant coefficient) be described by pure boundary integral equations. This approach has been extensively studied in the past, where methods of solution have been proposed handling the problem up to very high Péclet numbers [1,2].

To solve the problem of variable velocity, a decomposition of the velocity field into a constant and variable part has been proposed. The decomposition leads to a domain integral involving the variable part of the velocity field and the unknown field function. DeSilva et al. [3] used this approach to solve the transient conduction convection in 2-D. More recently, 
several authors solved the diffusion-convection equations with variable coefficients [4]. Decomposition to constant and variable part has been used here as well.

Ravnik and Skerget [5, 6] proposed an alternative approach, where the gradient of the coefficient is needed and gradient of the field function is not needed. Thus, the final integral equation includes only the unknown function on the boundary and in the domain and its flux on the boundary. The proposed equation is linear and after discretization requires only a single solution of a system of linear equations to obtain the solution. In this paper, we have applied this method for the solution of the energy transport equation with variable material properties.

\section{GOVERNING EQUATION}

We consider a domain $\Omega$ with a boundary $\Gamma$ filled with a fluid. Let $\vec{r} \in \Omega$ denote the position in the domain and let $t$ be the time. In the most general case, the fluid density, $\rho=\rho(\vec{r}, t)$, depends on time and location. If the fluid velocity field is denoted by $\vec{v}=\vec{v}(\vec{r}, t)$, the mass conservation law requires that

$$
\frac{\partial \rho}{\partial t}+\vec{\nabla} \cdot(\rho \vec{v})=0
$$

The energy equation for the fluid written with temperature $T(\vec{r}, t)$ as an independent variable is usually stated as

$$
\rho\left(\frac{\partial\left(c_{p} T\right)}{\partial t}+\vec{\nu} \cdot \vec{\nabla}\left(c_{p} T\right)\right)=\vec{\nabla} \cdot(k \vec{\nabla} T)+S,
$$

where the heat capacity $c_{p}=c_{p}(\vec{r}, t)$, thermal conductivity $k=k(\vec{r}, t)$ and domain heat sources $S(\vec{r}, t)$ all vary with time and location. Combining (1) and (2) one can show that the following is also true:

$$
\frac{\partial\left(\rho c_{p} T\right)}{\partial t}+\vec{\nabla} \cdot\left(\rho c_{p} T \vec{v}\right)=\vec{\nabla} \cdot(k \vec{\nabla} T)+S,
$$

This form of the energy transport equation is more convenient, as the density and heat capacity are joined in all terms, and can thus be treated as a single material property.

\section{DERIVATION}

As the governing equation is an unsteady diffusion-convection equation with sources, a fundamental solution, which would enable a boundary only representation of the equation does not exist. Due to the fact that the material properties and the velocity field are expected to vary with location and time, we employ the fundamental solution for the diffusion operator, since in this way the integrals, that will be needed, will not depend on time, material properties or the velocity field, and will have to be calculated only once for a chosen computational mesh. Thus, eqn (3) is multiplied by $u^{*}$, which is the fundamental solution for the diffusion operator

$$
u^{*}=\frac{1}{4 \pi|\vec{\xi}-\vec{r}|}
$$


and intergrated over the domain yielding

$$
\int_{\Omega} u * \vec{\nabla} \cdot(k \vec{\nabla} T) d \Omega=\int_{\Omega}\left(\frac{\partial\left(\rho c_{p} T\right)}{\partial t}+\vec{\nabla} \cdot\left(\rho c_{p} T \vec{v}\right)\right) u * d \Omega-\int_{\Omega} S u * d \Omega .
$$

Using the following vector algebra rule: $u^{*} \vec{\nabla} \cdot(k \vec{\nabla} T)=\vec{\nabla} \cdot\left(u^{*} k \vec{\nabla} T\right)-k \vec{\nabla} T \cdot \vec{\nabla} u^{*}$ we write

$$
\begin{aligned}
& \int_{\Omega} \vec{\nabla} \cdot\left(u^{*} k \vec{\nabla} T\right) d \Omega-\int_{\Omega} k \vec{\nabla} T \cdot \vec{\nabla} u * d \Omega \\
& =\int_{\Omega}\left(\frac{\partial\left(\rho c_{p} T\right)}{\partial t}+\vec{\nabla} \cdot\left(\rho c_{p} T \vec{v}\right)\right) u * d \Omega-\int_{\Omega} S u^{*} d \Omega .
\end{aligned}
$$

The first domain integral on the left hand side of (6) may be transformed into a boundary integral using the Gauss divergence theorem as

$$
\begin{aligned}
& \int_{\Gamma} u * k \vec{\nabla} T \cdot \vec{n} d \Gamma-\int_{\Omega} k \vec{\nabla} T \cdot \vec{\nabla} u * d \Omega \\
& \left.=\int_{\Omega} \frac{\partial\left(\rho c_{p} T\right)}{\partial t}+\vec{\nabla} \cdot\left(\rho c_{p} T \vec{v}\right)\right) u^{*} d \Omega-\int_{\Omega} S u^{*} d \Omega .
\end{aligned}
$$

Using $\vec{\nabla}(k T)=k \vec{\nabla} T+T \vec{\nabla} k$ we rewrite the second integral on the left hand side of (6) as

$$
\begin{aligned}
& \int_{\Gamma} u * k \vec{\nabla} T \cdot \vec{n} d \Gamma-\int_{\Omega} \vec{\nabla}(k T) \cdot \vec{\nabla} u * d \Omega+\int_{\Omega} T \vec{\nabla} k \cdot \vec{\nabla} u * d \Omega \\
& =\int_{\Omega}\left(\frac{\partial\left(\rho c_{p} T\right)}{\partial t}+\vec{\nabla} \cdot\left(\rho c_{p} T \vec{v}\right)\right) u^{*} d \Omega-\int_{\Omega} S u^{*} d \Omega .
\end{aligned}
$$

Using $\vec{\nabla} \cdot\left(k T \vec{\nabla} u^{*}\right)=\vec{\nabla}(k T) \cdot \vec{\nabla} u^{*}+k T \nabla^{2} u^{*}$ we obtain

$$
\begin{aligned}
& \int_{\Gamma} u^{*} k \vec{\nabla} T \cdot \vec{n} d \Gamma-\int_{\Omega} \vec{\nabla} \cdot\left(k T \vec{\nabla} u^{*}\right) d \Omega+\int_{\Omega} k T \nabla^{2} u^{*} d \Omega \\
& +\int_{\Omega} T \vec{\nabla} k \cdot \vec{\nabla} u * d \Omega=\int_{\Omega}\left(\frac{\partial\left(\rho c_{p} T\right)}{\partial t}+\vec{\nabla} \cdot\left(\rho c_{p} T \vec{v}\right)\right) u * d \Omega \\
& -\int_{\Omega} S u * d \Omega .
\end{aligned}
$$

The first domain integral on the left hand side may be transformed into a boundary integral using the Gauss divergence theorem as

$$
\begin{aligned}
& \int_{\Gamma} u * k \vec{\nabla} T \cdot \vec{n} d \Gamma-\int_{\Gamma} k \vec{\nabla} T u^{*} \cdot \vec{n} d \Gamma+\int_{\Omega} k T \nabla^{2} u^{*} d \Omega \\
& +\int_{\Omega} T \vec{\nabla} k \cdot \vec{\nabla} u^{*} d \Omega=\int_{\Omega}\left(\frac{\partial\left(\rho c_{p} T\right)}{\partial t}+\vec{\nabla} \cdot\left(\rho c_{p} T \vec{v}\right)\right) u^{*} d \Omega \\
& -\int_{\Omega} S u^{*} d \Omega .
\end{aligned}
$$


Since $u^{*}$ is the fundamental solution of the Laplace equation, the following integral equals $\int_{\Omega} k T \nabla^{2} u^{*} d \Omega=-k(\vec{\xi}) T(\vec{\xi})$ and we may write

$$
\begin{aligned}
& c(\vec{\xi}) k(\vec{\xi}) T(\vec{\xi})+\int_{\Gamma} k T \vec{\nabla} u^{*} \cdot \vec{n} d \Gamma=\int_{\Gamma} u * k \vec{\nabla} T \cdot \vec{n} d \Gamma \\
& +\int_{\Omega} T \vec{\nabla} k \cdot \vec{\nabla} u^{*} d \Omega-\int_{\Omega}\left(\frac{\partial\left(\rho c_{p} T\right)}{\partial t}+\vec{\nabla} \cdot\left(\rho c_{p} T \vec{v}\right)\right) u^{*} d \Omega \\
& +\int_{\Omega} S u^{*} d \Omega
\end{aligned}
$$

$c(\vec{\xi})$ is the geometric factor defined as $c(\vec{\xi})=\alpha / 4 \pi$, where $\alpha$ is the inner angle with origin in $\vec{\xi}$. The temperature at the boundary $T(\vec{r})$ or heat flux on the boundary $q(\vec{r})=\vec{\nabla} T(\vec{r}) \cdot \vec{n}$ are prescribed as boundary conditions.

Next, we used the following definition of divergence of a product

$$
u^{*} \vec{\nabla} \cdot\left(\rho c_{p} T \vec{v}\right)=\vec{\nabla} \cdot\left(u^{*} \rho c_{p} T \vec{v}\right)-\rho c_{p} T \vec{v} \cdot \vec{\nabla} u^{*}
$$

to arrive at

$$
\begin{aligned}
& c(\vec{\xi}) k(\vec{\xi}) T(\vec{\xi})+\int_{\Gamma} k T \vec{\nabla} u^{*} \cdot \vec{n} d \Gamma=\int_{\Gamma} u^{*} k \vec{\nabla} T \cdot \vec{n} d \Gamma \\
& -\int_{\Omega} \vec{\nabla} \cdot\left(\rho c_{p} \vec{v} u^{*} T\right) d \Omega+\int_{\Omega} T\left(\vec{\nabla} k+\rho c_{p} \vec{v}\right) \cdot \vec{\nabla} u^{*} d \Omega \\
& -\int_{\Omega} \frac{\partial\left(\rho c_{p} T\right)}{\partial t} u^{*} d \Omega+\int_{\Omega} S u^{*} d \Omega
\end{aligned}
$$

The first domain integral on the right hand side may be transformed into a boundary integral using the Gauss divergence theorem as

$$
\begin{aligned}
& c(\vec{\xi}) k(\vec{\xi}) T(\vec{\xi})+\int_{\Gamma} k T \vec{\nabla} u * \cdot \vec{n} d \Gamma=\int_{\Gamma} u^{*} k \vec{\nabla} T \cdot \vec{n} d \Gamma \\
& -\int_{\Gamma} \rho c_{p} u^{*} T \vec{v} \cdot \vec{n} d \Gamma+\int_{\Omega} T\left(\vec{\nabla} k+\rho c_{p} \vec{v}\right) \cdot \vec{\nabla} u^{*} d \Omega \\
& -\int_{\Omega} \frac{\partial\left(\rho c_{p} T\right)}{\partial t} u^{*} d \Omega+\int_{\Omega} S u^{*} d \Omega .
\end{aligned}
$$

The time derivative term is separated by

$$
\frac{\partial\left(\rho c_{p} T\right)}{\partial t}=\rho c_{p} \frac{\partial T}{\partial t}+T \frac{\partial \rho c_{p}}{\partial t}
$$

to yield the final boundary-domain integral form of the energy equation with variable material properties: 


$$
\begin{aligned}
& c(\vec{\xi}) k(\vec{\xi}) T(\vec{\xi})+\int_{\Gamma} k T \vec{\nabla} u^{*} \cdot \vec{n} d \Gamma=\int_{\Gamma} u^{*} k \vec{\nabla} T \cdot \vec{n} d \Gamma \\
& -\int_{\Gamma} \rho c_{p} u^{*} T \vec{v} \cdot \vec{n} d \Gamma+\int_{\Omega} T\left(\vec{\nabla} k+\rho c_{p} \vec{v}\right) \cdot \vec{\nabla} u^{*} d \Omega \\
& -\int_{\Omega}\left(\rho c_{p} \frac{\partial T}{\partial t}+T \frac{\partial\left(\rho c_{p}\right)}{\partial t}\right) u^{*} d \Omega+\int_{\Omega} S u^{*} d \Omega .
\end{aligned}
$$

\section{DISCRETIZATION}

At time $t$ for a time step $\Delta t$ the backward Euler finite difference approximation is used to approximate the time derivative as

$$
\frac{\partial T}{\partial t}=\beta_{1} T+\beta_{2} T^{c}+\beta_{3} T^{p}
$$

where $\beta_{1}=\frac{3}{2 \Delta t}, \beta_{2}=-\frac{2}{\Delta t}$ and $\beta_{3}=\frac{1}{2 \Delta t} . T$ is the temperature in the next time step, $T^{c}$ is the temperature in the current time step and $T^{p}$ is the temperature in the previous time step. Using (15) in (13), we get

$$
\begin{aligned}
& c(\vec{\xi}) k(\vec{\xi}) T(\vec{\xi})+\int_{\Gamma} k T \vec{\nabla} u^{*} \cdot \vec{n} d \Gamma=\int_{\Gamma} u^{*} k \vec{\nabla} T \cdot \vec{n} d \Gamma \\
& -\int_{\Gamma} \rho c_{p} u^{*} T \vec{v} \cdot \vec{n} d T+\int_{\Omega} T\left(\vec{\nabla} k+\rho c_{p} \vec{v}\right) \cdot \vec{\nabla} u^{*} d \Omega \\
& -\int_{\Omega}\left(\rho c_{p}\left(\beta_{1} T+\beta_{2} T^{c}+\beta_{3} T^{p}\right)+T \frac{\partial\left(\rho c_{p}\right)}{\partial t}-S\right) u^{*} d \Omega
\end{aligned}
$$

We use domain decomposition to write the discrete system of equations. We make a mesh of the entire domain $\Omega$ and name each mesh element a subdomain. Then eqn (16) is written for each of the subdomains. We use shape functions to interpolate field functions and flux across the boundary and inside of the subdomain. A function, e.g., temperature, is interpolated over a boundary elements as $T=\sum \varphi_{i} T_{i}$, inside each subdomain as $T=\sum \Phi_{i} T_{i}$, while flux is interpolated over boundary elements as $q=\sum \phi_{i} q_{i}$. The following integrals must be calculated:

$$
\begin{gathered}
{[H]=\int_{\Gamma} \varphi_{i} \vec{\nabla} u^{*} \cdot \vec{n} d \Gamma, \quad[G]=\int_{\Gamma} \phi_{i} u^{*} d \Gamma,} \\
{[\vec{A}]=\int_{\Gamma} \varphi_{i} \vec{n} u^{*} d \Gamma, \quad[\vec{D}]=\int_{\Omega} \Phi_{i} \vec{\nabla} u^{*} \Omega, \quad[B]=\int_{\Omega} \Phi_{i} u^{*} d \Omega .}
\end{gathered}
$$

The square brackets denote integral matrices. Each source point location yields one row in these matrices. The source point is set to all function and flux node in each subdomain. By letting curly brackets denote vectors of nodal values of field functions, we may write the discrete energy equation as:

$$
\begin{gathered}
{[H]\{k\}\{T\}=[G]\{k\}\{q\}-\left(\left[A_{x}\right]\left\{\rho c_{p} v_{x}\right\}+\left[A_{y}\right]\left\{\rho c_{p} v_{y}\right\}+\left[A_{z}\right]\left\{\rho c_{p} v_{z}\right\}\right)\{T\}} \\
+\left(\left[D_{x}\right]\left\{\frac{\partial k}{\partial x}+\rho c_{p} v_{x}\right\}+\left[D_{y}\right]\left\{\frac{\partial k}{\partial y}+\rho c_{p} v_{y}\right\}+\left[D_{z}\right]\left\{\frac{\partial k}{\partial y}+\rho c_{p} v_{z}\right\}\right)\{T\} \\
-[B]\left\{\rho c_{p} \beta_{1}+\frac{\partial\left(\rho c_{p}\right)}{\partial t}\right\}\{T\}-[B]\left\{\rho c_{p} \beta_{2} T^{c}+\rho c_{p} \beta_{3} T^{p}\right\}+[B]\{S\}
\end{gathered}
$$


The terms involving the unknown temperature values $\{T\}$ may be summed up (in node by node sense) to form:

$$
\begin{array}{r}
\left([H]\{k\}+\left[A_{x}\right]\left\{\rho c_{p} v_{x}\right\}+\left[A_{y}\right]\left\{\rho c_{p} v_{y}\right\}+\left[A_{z}\right]\left\{\rho c_{p} v_{z}\right\}\right. \\
-\left[D_{x}\right]\left\{\frac{\partial k}{\partial x}+\rho c_{p} v_{x}\right\}-\left[D_{y}\right]\left\{\frac{\partial k}{\partial y}+\rho c_{p} v_{y}\right\} \\
\left.-\left[D_{z}\right]\left\{\frac{\partial k}{\partial z}+\rho c_{p} v_{z}\right\}+[B]\left\{\rho c_{p} \beta_{1}+\frac{\partial\left(\rho c_{p}\right)}{\partial t}\right\}\right)\{T\} \\
=[G]\{k\}\{q\}+[B]\left\{S-\rho c_{p} \beta_{2} T^{c}-\rho c_{p} \beta_{3} T^{p}\right\}
\end{array}
$$

The final step involves taking note of the boundary conditions and setting up the system matrix accordingly. Either temperature $\{\mathrm{T}\}$ or flux $\{\mathrm{q}\}$ may be prescribed. The final system is linear as long as the material properties, flow velocity and sources do not depend on temperature. If they do, an iterative scheme must be set up which involves repeated solutions of the system along with updating of the material properties, flow velocity and sources.

\section{TEST CASES}

In order to verify the validity of the derivation and implementation, we performed extensive tests. We chose a cubical domain $(0,0,0) \times(1,1,1)$ where an incompressible fluid flows with a velocity field $\vec{v}=(x,-2 y, z)$. We consider two cases, $A$ and $B$, which both feature variable material properties in the domain. Additional parameter, $\mathbf{K}$, was introduced to adjust the magnitude of variation of material properties. Heat sources were added to the domain so that the resulting temperature solution was $T_{a}=x y z t$. Material parameters and sources are listed in Table 1 .

A combination of Dirichlet and Neumann boundary conditions was used. Two opposite facing walls had a known temperature prescribed, while the other four walls of the cubical domain had a known heat flux prescribed. The domain and the boundary conditions are shown in Fig. 1. The temperature field was initialized using the analytical solution at $t=0$ as $T=0$. The solution was advanced through time using a time step of $\Delta t=0.01$ until $t=1$, when the results are analyzed.

The meshes chosen to solve the two tests had $2^{3}, 4^{3}, 8^{3}, 16^{3}, 32^{3}$ hexahedral elements with $5^{3}, 9^{3}, 17^{3}, 33^{3}, 65^{3}$ nodes. The nodes were placed equidistantly in the domain, so the element sizes were $h=0.200,0.111,0.058,0.030,0.015$.

In order to compare the simulation results with analytical values, a root- mean-square norm,

$$
\left\|T_{h}-T_{a}\right\|=\left(\frac{\sum_{i=1}^{n}\left(T_{h, i}-T_{a, i}\right)^{2}}{\sum_{i=1}^{n} T_{a, i}^{2}}\right)^{1 / 2}
$$

Table 1: Material properties and source for the test cases solved. An additional parameter $k$ is used to increase the severity of spatial and temporal changes in material parameters. $\kappa=1,2,4$ were considered.

\begin{tabular}{lccc}
\hline case & $\rho c_{p}$ & $k$ & $S$ \\
\hline$A$ & 1 & $2+\cos (\kappa \pi x y z)$ & $x y z+\pi t\left(y^{2} z^{2}+x^{2}\left(y^{2}+z^{2}\right)\right) \kappa \sin (\pi x y z \kappa)$ \\
$B$ & $1+x^{\kappa}$ & $1+z^{\kappa}$ & $x y\left(-t z^{\kappa-1} \kappa+z\left(1+x^{\kappa}(1+t \kappa)\right)\right)$ \\
\hline
\end{tabular}




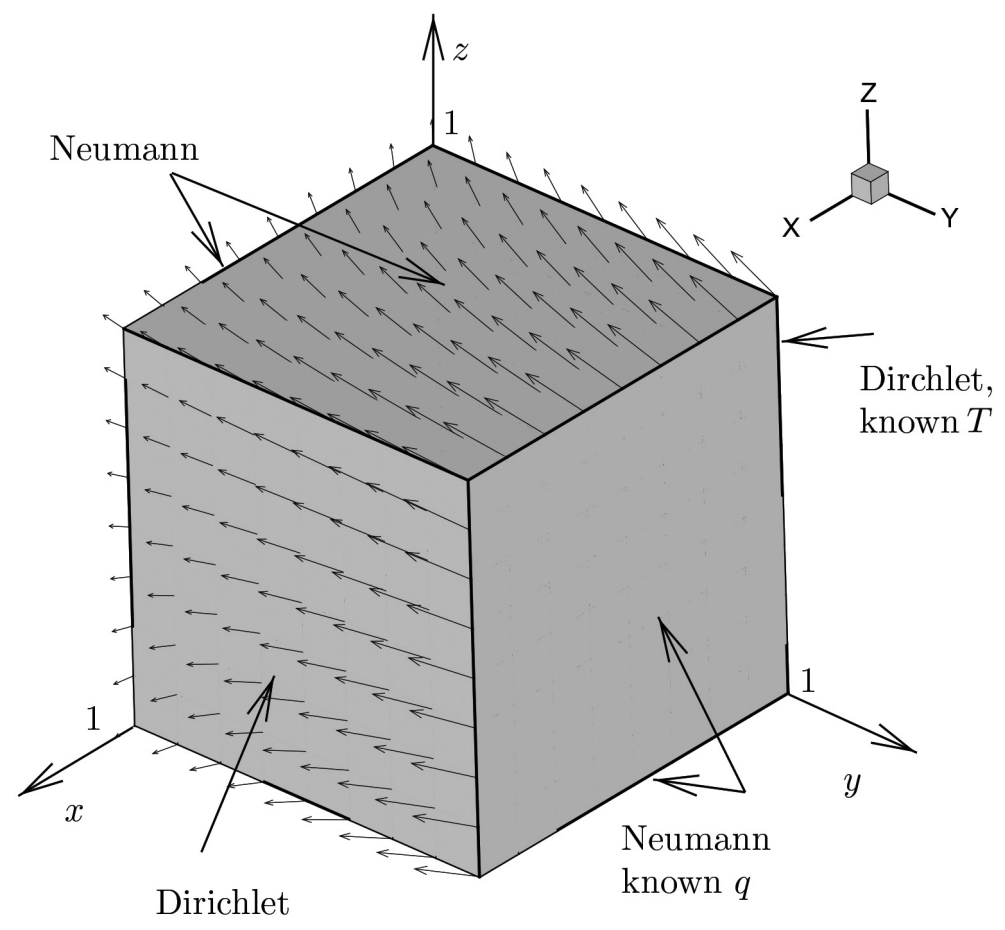

Figure 1: The domain and a vector based visualization of the flow field. Boundary conditions are also shown.

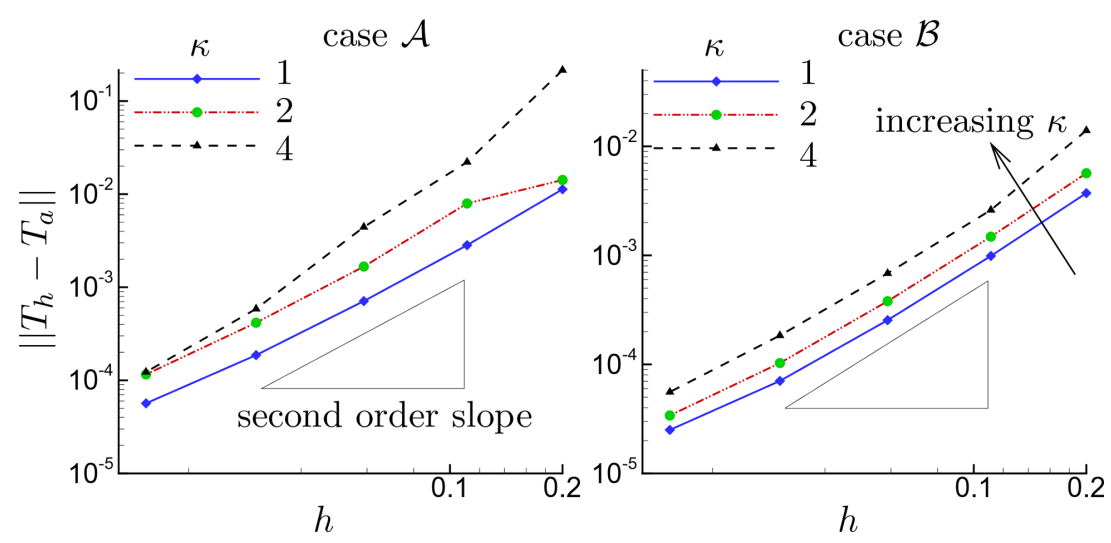

Figure 2: Norms expressing the difference between simulation and analytical results. Secondorder convergence slope is also shown.

has been used. Here, $i$ sums values from nodes inside of the domain (boundary conditions are skipped) so $\mathrm{T}_{\mathrm{h}, \mathrm{i}}$ are the nodal values obtained on a mesh with element size $h$.

The norms for both test cases are shown in Fig. 2. We observe good convergence of results, accuracy increases when element size decreases. Three values of the parameter $\kappa=1,2,4$ were used. Since larger $k$ corresponds to stronger spatial and temporal variation of material parameters, we observe lowering of accuracy with increasing $\boldsymbol{K}$. 
Since the element sizes decrease by a factor of 2 it is possible to employ the Richardson extrapolation to estimate the order of the proposed method. The method's order is defined as

$$
O=\frac{1}{\log 2} \log \left(\frac{\left\|T_{h / 2}-T_{a}\right\|}{\left\|T_{h}-T_{a}\right\|}\right) .
$$

A slope, which corresponds to second-order accuracy, is shown in Fig. 2. We observe that the norms for both cases exhibit convergence slopes which are comparable to the second-order slope. Equation (21) was used to calculate the method's order using all available results. We obtained $\mathrm{O}=1.996 \pm 0.46$, which clearly indicates that the developed method is second-order accurate.

\section{APPLICATION TO NANOFLUID SIMULATION}

A nanofluid is a stable suspension of nanometer-sized particles suspended in a fluid. A typical application of nanofluid is an enhancement of heat transfer, thus particles are usually metal oxides having better heat transfer properties than the base fluid.

Consider nanoparticles distributed in the fluid. Local concentration of nanoparticles is

$$
c(\vec{r})=\frac{N(\vec{r})}{V(\vec{r})},
$$

where $N(\vec{r})$ is the number of nanoparticles in a local volume $V(\vec{r})$ around a location $\vec{r}$. As nanoparticles are extremely small, it is impossible to simulate a number large enough to account for the actual concentration. Thus, a small number of particles it tracked (e.g. $10^{5}$ ), which represent a fraction of the bulk volume fraction.

We estimate the particle volume fraction as

$$
\varphi(\vec{r})=\varphi_{0}+\varphi^{\prime}\left(\frac{c(\vec{r})}{\frac{1}{V} \int_{V} c(\vec{r}) d V}-1\right),
$$

where $\varphi_{0}$ is the bulk solid nanoparticle volume fraction and $\varphi^{\prime}$ is the magnitude of an oscillating part, which is estimated by Lagrangian tracking of individual particles.

As the flow field transports the particles the changes in the local volume fraction are reflected in spatial and temporal changes of heat conductivity, heat capacity, and density of the nanofluid. In order to properly estimate the heat transport, a transport equation with variable material properties, such as eqn (3) must be solved.

Nanoparticles have a very low Stokes number, thus they follow the fluid flow closely. However, due to their small size they also exhibit Brownian motion due to constant molecular bombardment. Moreover, in cases where the temperature gradient is present, the higher momentum of warmer fluid molecules giver rise to the thermophoresis effect. Thermophoresis is the result of averaged Brownian motion in a fluid.

The thermophoresis effect has been studied in gases by Epstein [7] who developed a model for the average velocity of the particle due to thermophoresis. This was corrected for the use in liquids by McNab and Meisen [8], who have shown, that thermophoresis is slower in liquids. Recently, Michaelides [9] realized that thermophoretic velocity depends strongly on fluid and nanoparticles type, so he performed Monte-Carlo simulations to provide estimates for several common nanofluids. According to this results aluminum oxide-water nanofluid has the following thermophoretic velocity 


$$
\vec{v}_{t p}=-1264\left(\frac{r}{r_{0}}\right)^{-1.417} \frac{\mu_{f}}{\rho_{f}} \frac{\vec{\nabla} T}{T},
$$

where $r$ is the nanoparticle radius and $r_{0}=1 \mathrm{~nm}$. The thermophoretic force is then calculated by $\vec{F}_{t b}=3 \pi \mu_{f} d \vec{v}_{t b}$. Finally, the movement of particles may be estimated by

$$
\vec{r}(t+\Delta t)=\vec{r}(t)+\left(\vec{v}_{f}(\vec{r})+\vec{v}_{t p}\right) \Delta t,
$$

where $\vec{v}_{f}(\vec{r})$ is the velocity of the fluid at the position of the nanoparticle.

The solution of the energy equation and the Lagrangian particle tracking were included in our in-house BEM-based nanofluid flow and heat transfer solver [10]. A standard natural

Table 2: Simulation of heat transfer of $\mathrm{Al}_{2} \mathrm{O}_{3}$ nanofluid in a 3D cubic cavity with differential heated walls. Heat transfer through a heated wall is shown expressed with a Nusselt number as a function of the Rayleigh number $(R a)$. Average solid particle volume fraction was $\varphi_{0}=0.1$. In the study [11] nanofluid properties were estimated using the average solid particle volume fraction and were assumed constant in space and time.

\begin{tabular}{lrllll}
\hline$R a$ & {$[11]$} & Present & $R a$ & {$[11]$} & Present \\
\hline $10^{3}$ & 1.345 & 1.345 & $10^{5}$ & 4.806 & 4.905 \\
$10^{4}$ & 2.168 & 2.179 & $10^{6}$ & 9.817 & 10.15 \\
\hline
\end{tabular}

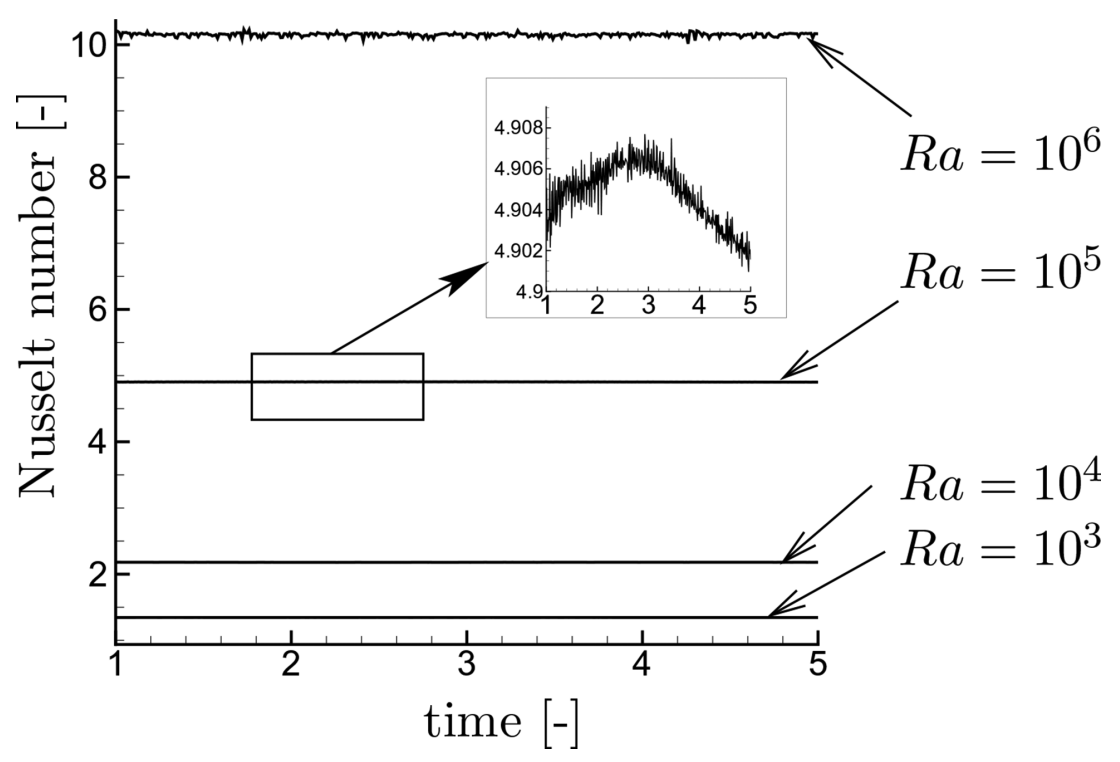

Figure 3: Heat transfer though a vertical wall expressed as Nusselt number versus time for four different Rayleigh numbers. 
convection test case was solved-an insulated cubic cavity with two opposite walls heated and cooled [11] using a $17^{3}$ node mesh. $\mathrm{A} \mathrm{Al}_{2} \mathrm{O}_{3}$ nanofluid was considered.

Table 2 shows the comparison of the time averaged heat fluxes and Fig. 3 shows time traces of heat flux. While looking at the time traces, we observe small oscillations of heat flux due to variations in nanofluid properties. The oscillations increase in magnitude as the Rayleigh number is increased. This is due to the fact that bouyant forces increase with Rayleigh number, and thus the flow field in the cavity is more complex which in turn results in higher variation of particle concentration. The time average values of the Nusselt match reference results for low Rayleigh numbers, while at higher Rayleigh number values, the variations in nanofluid properties yield a slightly lower heat transfer rates.

\section{SUMMARY}

The paper presents a boundary element based numerical method for the solution of the energy transport equation, when the fluid properties (density, specific heat, thermal conductivity) exhibit spatial and temporal variations. Examples of such fluids are nanofluids, which are stable suspensions of nanoparticles in a base fluid. Since the concentration of nanoparticles varies with the fluid flow, the average properties of a nanofluid, when modeled as a single phase, vary with location and time.

The derived boundary-domain integral formulation (eqn 14) does not include the gradient of the temperature field. For a known flow velocity field, this enables finding the unknown temperature field using a single solution of the discrete system of linear equations. The proposed method was verified by analytical test cases and was found to be second-order accurate. Furthermore, we used the developed method in a Lagrange-Euler model for nanofluid simulation.

\section{REFERENCES}

[1] Skerget, L., Zagar, I. \& Alujevic, A., Three-dimensional steady-state diffusion-convection. In Boundary Elements IX, 3, Springer: Berlin, 1987.

[2] Qiu, Z.H., Wrobel, L. \& Power, H., Numerical solution of convection-diffusion problems at high Peclet number using boundary elements. International Journal for Numerical Methods in Engineering, 41, pp. 899-914, 1998. http://dx.doi.org/10.1002/(SICI)1097-0207(19980315)41:5<899::AID-NME314>3.0.CO;2-T

[3] DeSilva, S.J., Chan, C.L., Chandra, A. \& Lim, J., Boundary element method analysis for the transient conduction convection in 2-D with spatially variable convective velocity. Applied Mathematical Modelling, 22(12), pp. 81-112, 1998. http://dx.doi.org/10.1016/S0307-904X(98)00010-9

[4] Rap, A., Elliott, L., Ingham, D.B., Lesnic, D. \& Wen, X., DRBEM for Cauchy convection-diffusion problems with variable coefficients. Engineering Analysis with Boundary Elements, 28(11), pp. 1321-1333, 2004. http://dx.doi.org/10.1016/j.enganabound.2004.06.003

[5] Ravnik, J. \& Skerget, L., A gradient free integral equation for diffusion - convection equation with variable coefficient and velocity. Engineering Analysis with Boundary Elements, 37, pp. 683-690, 2013. http://dx.doi.org/10.1016/j.enganabound.2013.01.012

[6] Ravnik, J. \& Skerget, L., Integral equation formulation of an unsteady diffusionconvection equation with variable coefficient and velocity. Computers and Mathematics with Applications, 66(12), pp. 2477-2488, 2014. http://dx.doi.org/10.1016/j.enganabound.2013.01.012 
[7] Epstein, P., Zur theorie des radiometers. Zeitschrift fur Physik, 54, pp. 537-563, 1929. http://dx.doi.org/10.1007/BF01338485

[8] McNab, G.S. \& Meisen, A., Thermophoresis in liquids. Journal of Colloid and Interface Science, 44(2), pp. 339-346, 1973. http://dx.doi.org/10.1016/0021-9797(73)90225-7

[9] Michaelides, E.E., Brownian movement and thermophoresis of nanoparticles in liquids. International Journal of Heat and Mass Transfer, 81, pp. 179-187, 2015. http://dx.doi.org/10.1016/j.ijheatmasstransfer.2014.10.019

[10] Ravnik, J. \& Skerget, L., A numerical study of nanofluid natural convection in a cubic enclosure with a circular and an ellipsoidal cylinder. International Journal of Heat and Mass Transfer, 89, pp. 596-605, 2015.

http://dx.doi.org/10.1016/j.ijheatmasstransfer.2015.05.089

[11] Ravnik, J., Skerget, J. \& Hribersek, M., Analysis of three-dimensional natural convection of nanofluids by BEM. Engineering Analysis with Boundary Elements, 34, pp. 1018-1030, 2010.

http://dx.doi.org/10.1016/j.enganabound.2010.06.019 